\title{
P20-16. Ultra-deep pyrosequencing detects complex patterns of CD8+ T-lymphocyte escape in SIV-infected macaques
} BJ Burwitz*1, BN Bimber'2, S O'Connor ${ }^{1}$, A Detmer ${ }^{2}$, E Gostick ${ }^{3}$, SM Lank², DA Price ${ }^{3}$, A Hughes ${ }^{4}$ and D O'Connor ${ }^{1}$

Address: ${ }^{1}$ Cellular and Molecular Pathology, University of Wisconsin-Madison, Madison, WI, USA, ${ }^{2}$ Wisconsin National Primate Research Center, Madison, WI, USA, ${ }^{3}$ Cardiff University School of Medicine, Cardiff, UK and ${ }^{4}$ University of South Carolina, Columbia, SC, USA

* Corresponding author

from AIDS Vaccine 2009

Paris, France. 19-22 October 2009

Published: 22 October 2009

Retrovirology 2009, 6(Suppl 3):P386 doi:10.1 186/1742-4690-6-S3-P386

This abstract is available from: http://www.retrovirology.com/content/6/S3/P386

(c) 2009 Burwitz et al; licensee BioMed Central Ltd.

\section{Background}

A complex population of viral variants exists within each individual infected with immunodeficiency virus. Deciphering the breadth and frequency of accruing viral mutations provides insight into immune responses, drug resistance, and potential vaccine targets. Contemporary sequencing methods are limited to detection of high frequency variants, leading to an incomplete assessment of the overall viral population. Here, we use ultra-deep pyrosequencing to create a comprehensive picture of CD8+ Tlymphocyte (CD8-TL) escape in two epitopes in SIVinfected rhesus and cynomolgus macaques, revealing a complex pattern of viral variants previously undetected.

\section{Methods}

Plasma was collected from SIV-infected rhesus and cynomolgus macaques at multiple timepoints between weeks 1 and 20 post-infection. Viral RNA was isolated and amplicons spanning the epitopes of interest were generated by RT-PCR, using primers that incorporated a unique 10 bp molecular barcode into each sample. Amplicons were pooled and sequenced on a Roche Genome Sequencer FLX instrument and analyzed using Roche Amplicon Variant Analyzer software.

\section{Results}

The increased sensitivity of ultra-deep pyrosequencing enabled detection of acute CD8-TL escape as early as 17 days post-infection, representing the earliest published example of CD8-TL escape in intrarectally infected macaques. Conversely, we observed the continued presence of a complex viral population well into chronic infection, indicating that viral mutations deemed "fixed" by Sanger sequencing are instead complemented by a broad array of viral variants. Additionally, we show that these methods can be applied to sequencing of the entire SIVmac239 genome, supporting the continued use of pyrosequencing in comprehensive SIV infection studies.

\section{Conclusion}

Overall, these findings demonstrate that pyrosequencing can be used to study viral evolution during HIV/SIV infection with an unprecedented degree of sensitivity. Utilizing newly emerging molecular tools is essential and will further our understanding of how viral pathogens evade the immune system. 